\title{
Matrin 3 Augments the Transcriptional Activity of an SV40 Promoter-Mediated Luciferase Gene with a Highly Repetitive DNA Component
}

Shinya Kamiuchi ${ }^{1}$, Mutsumi Fukaya ${ }^{1}$, Tatsuhiro Usui ${ }^{1}$, Naohiro Iwata ${ }^{1}$, Mari Okazaki ${ }^{2}$, Hirokazu Matsuzaki ${ }^{2}$, Katsuyoshi Sunaga ${ }^{1}$ and Yasuhide Hibino $^{{ }^{*}}$

${ }^{1}$ Laboratory of Immunobiochemistry, Department of Clinical Dietetics \& Human Nutrition, Faculty of Pharmaceutical Sciences, Josai University, Japan

${ }^{2}$ Laboratory of Pharmacology, School of Pharmaceutical Sciences, Faculty of Pharmaceutical Sciences, Josai University, Japan

*Corresponding author: Yasuhide Hibino, Laboratory of Immunobiochemistry, Department of Clinical Dietetics and Human Nutrition, Faculty of Pharmaceutical Sciences, Josai University, 1-1 Keyakidai Sakado, Saitama 350-0295, Japan, Tel: +81 49 271 7285; Fax: +81 49 271 7284; E-mail: seitaib@josai.ac.jp

Received date: September 29, 2014, Accepted date: November 20, 2014, Published date: November 28, 2014

Copyright: (C) 2014 Kamiuchi S, et al. This is an open-access article distributed under the terms of the Creative Commons Attribution License, which permits unrestricted use, distribution, and reproduction in any medium, provided the original author and source are credited.

\begin{abstract}
We examined the transcriptional augmentation of matrin 3, a nuclear matrix protein, of the SV40 promotermediated luciferase gene (pGL3) following transient transfection of recombinant plasmids into cells. It has been reported that the interaction of the Xmn I fragment, a highly repetitive DNA component as one of a typical matrix- or scaffold-attachment regions (MAR/SAR) tethered upstream from the SV40 promoter (pGL3-Xmn I) with matrin 3 appeared to be required for augmentation of luciferase gene transcription. In this study, we investigated the levels of induction in cells overexpressing the wild type and several deletion mutants of matrin 3. It appeared that pGL3-Xmn I augmented luciferase production to 4-times the control level in Ac2F cells, but 23-fold in cells overexpressing matrin 3. Electrophoretic mobility shift assay showed that the $X m n$ I fragment augmented luciferase gene transcription through interaction with matrin 3. Furthermore, our findings suggest that all of the functional domains tested in matrin 3 were necessary for transcriptional augmentation. We aim not only to describe the transcriptional augmentation of matrin 3 with MAR/SAR, but also to strengthen interest in their use to mediate the expression of therapeutic transgenes.
\end{abstract}

Keywords: Matrin 3; Nuclear matrix; Matrix attachment regions; Highly repetitive DNA; Therapeutic transgene

\section{Introduction}

The nuclear matrix or scaffold was initially identified as a structural framework of nuclear proteins that can be represented by fibrous structures extending from the entire surface of denatured chromosomes [1]. DNA segments to which matrix or scaffold proteins can bind are termed matrix- or scaffold-attachment regions (MAR/ SAR). Structural analyses of the nucleus and chromosomes assigned a significant role of MAR/SAR to the matrix or scaffold-mediated stabilization of chromosomal structure. In the interphase cell nucleus, chromatin is arranged in spatially separate, chromosome-specific territories, which are maintained intact on the nuclear matrix as a three-dimensional organization of the chromosomes [2]. The organized structure of chromosomes is likely modulated in association with transcription, replication, RNA splicing, and the cell-division cycle, although mechanisms for the preferential alterations in chromosomal structure are as yet largely unknown.

Several MAR/SAR binding proteins have been identified. In our previous work, a 4.6-kb long P130 cDNA (AB205483) was cloned to determine the molecular properties of P130 [3]. P130, comprising 845 amino acid residues and having molecular mass of $95.3 \mathrm{kDa}$, was identical in structure to rat matrin 3 (BC062231) as revised from that published by Belgrader et al. [4], and highly similar to human and mouse matrin 3 (BC015031 and BC090833, respectively). Matrin 3 has several functionally confirmed motifs such as a nuclear export signal (NES), a nuclear localization signal (NLS), $\mathrm{C}_{2} \mathrm{H}_{2}$-type zinc-finger motifs (ZF1 and ZF2), RNA-binding domains (RB1 and RB2) and a membrane retention signal (MRS).
In vitro DNA-binding assays performed by us suggested several unique properties of matrin 3 . It bound to a highly repetitive DNA component, an Xmn I fragment, which was also cloned from DNA contained in rat liver nuclear scaffold [5]. In addition to binding to the Xmn I fragment, matrin 3 bound to various MAR/SAR segments by recognizing an ATATAT sequence, which causes base-unpairing [6,7], as a binding site. Functionally, transient expression of reporter luciferase gene constructs, in which Xmn I or distinct MAR/SAR fragments were tethered upstream from the SV40 promoter of the pGL3 promoter vector, increased luciferase gene transcription in various types of recipient cells [7]. Bent and AT-rich regions of Xmn I and MAR/SAR fragments appeared to be required for this increased transcription. Southwestern blot analysis revealed that an Xmn I probe bound to a $130-\mathrm{kDa}$ polypeptide in a nuclear extract from Ac2F cells, suggests that the binding of P130 as matrin 3 to an appropriate sequence positioned near the promoter of a gene likely modulates transcriptional activity.

In the present study, using several deletion mutants of matrin 3 expressed in Ac2F cells, we examined the levels of induction in cells overexpressing the wild type with the aim of determining which domain in matrin 3 is necessary for reporter luciferase transcriptional augmentation of the Xmn I fragment tethered upstream from the SV40 promoter.

\section{Materials and Methods}

\section{Cell line}

The rat hepatoma cell line Ac2F was provided by the Japanese Cancer Research resource and maintained in monolayer culture with minimum essential medium (MEM) containing $10 \%$ fetal bovine 
Page 2 of 5

serum and $0.006 \%$ kanamycin under $5 \% \mathrm{CO}_{2}$ and $95 \%$ air at $37^{\circ} \mathrm{C}$ Culture media (Life Technologies, Carlsbad, CA, USA) and all other materials were purchased through local distributors.

\section{Plasmids carrying the Xmn I fragment and wild-type or deletion mutants of matrin 3}

Promoter vector pGL3 (Promega Corp, Madison, WI, USA) was used to determine the extent of augmentation of SV40 promotermediated luciferase gene transcription by the $\mathrm{Xmn}$ I fragment tethered up-stream from the tract encompassing the SV40 promoter and the firefly luciferase gene [7].

cDNA encoding wild-type or deletion mutants of matrin 3 were prepared by ligating the PCR-amplified product and subcloning into pcDNA [3]. Plasmid DNA was propagated in E. coli DH5a and the final preparation was obtained by $\mathrm{CsCl}$ equilibrium centrifugation. Nucleotide sequences of DNA constructs tested were confirmed.

\section{Transfection and expression in stable transfectants}

Matrin 3 and its deletion mutants were expressed in stable transfectants generated from Ac2F cells by electroporation of the recombinant plasmids. These cells were incubated and maintained in $\mathrm{MEM}$ at $37^{\circ} \mathrm{C}$ under $5 \% \mathrm{CO}_{2}$ and $95 \%$ air containing G418 (400 $\mu \mathrm{g} /$ $\mathrm{ml})$.

\section{Determination of the extent of augmentation of transcription}

Lipofection of the recombinant pGL3 and pRL-SV40 with Transfectam (Promega Corp) into cells subcultured in dishes with a diameter of $60 \mathrm{~mm}$, preparation of cell lysate, and determination of luciferase activity using a dual luciferase assay kit on a TopCount (Packard Instrument Co., Inc., Meriden, CT, USA) were performed according to the supplier's instructions. The extent of augmentation of transcription was determined as luciferase activity exhibited by cells that received wild type pGL3 promoter vector and pRL-SV40 [7].

\section{Preparation of the ${ }^{32} \mathrm{P}$-labeled Xmn I fragment and electrophoretic mobility shift assay (EMSA)}

The 370-bp Xmn I fragments were labeled with $\left[{ }^{32} \mathrm{P}\right] \mathrm{dCTP}$ (Bresatec) in a T4 DNA polymerase labeling system (Life Technologies), according to the methods described in instruction manuals from the suppliers [8]. The resulting ${ }^{32} \mathrm{P}$-labeled DNA was designated as the $\left.{ }^{32} \mathrm{P}\right] \mathrm{Xmn}$ I fragment. EMSA was performed using the $\left[{ }^{32} \mathrm{P}\right] X m n$ I fragment with nuclear extract in a final volume of 25 $\mu \mathrm{l}$ of reaction mixture containing $10 \mathrm{mM}$ Tris- $\mathrm{HCl}$ ( $\mathrm{pH}$ 7.4), $40 \mathrm{mM}$ $\mathrm{NaCl}, 1 \mathrm{mM}$ EDTA, 4\% glycerol, and $1 \mathrm{mM}$ 2-mercaptoethanol. Nuclear extracts were prepared from Ac2F and Ac2F highly expressing matrin 3 [3]. In brief, all the mixtures were incubated at room temperature for $30 \mathrm{~min}$. The incubated mixtures were directly electrophoresed on a slab gel [ $4 \%$ polyacrylamide, $7.6 \mathrm{mM}$ Tris- $\mathrm{HCl}$ (pH 7.9), $3.3 \mathrm{mM}$ sodium acetate, $1 \mathrm{mM}$ EDTA] at $4^{\circ} \mathrm{C}$ for $2.5 \mathrm{~h}$ at 200 $\mathrm{V}$ and subjected to an autoradiographic assay. The $\left[{ }^{32} \mathrm{P}\right] X m n \mathrm{I}$ fragment complexes were quantified using a BAS2000 imaging analyzer (Fujifilm, Tokyo, Japan).

\section{Western blot analysis}

Whole cell extracts were homogenized in SDS sample buffer containing $125 \mathrm{mM}$ Tris- $\mathrm{HCl}$ (pH 6.8), 4\% SDS, $10 \%$ sucrose, $0.01 \%$ bromophenol blue, and 10\% 2-mercaptoethanol and boiled for $1 \mathrm{~min}$. Protein concentration was quantified by using the Bradford method (Protein Assay Reagent Kit, Bio-Rad Laboratories, Inc., Hercules, CA, USA). The samples were separated by SDS-polyacrylamide gel electrophoresis, transferred onto nitrocellulose membrane (GE Healthcare, Uppsala, Sweden) through a semidry-type blotting (BioRad Laboratories) method, blocked by $5 \%$ nonfat dry milk in PBS with Tween-20 (PBS-T) (137 mM NaCl, $8.10 \mathrm{mM} \mathrm{Na}_{2} \mathrm{HPO}_{4}, 2.68 \mathrm{mM} \mathrm{KCl}$, $1.47 \mathrm{mM} \mathrm{KH}_{2} \mathrm{PO}_{4}, 0.1 \%$ Tween-20), and incubated with appropriate antibodies as described below. The filters were incubated with each primary antibody for overnight at $4^{\circ} \mathrm{C}$, with the corresponding horseradish peroxidase (HRP)-conjugated secondary antibody for $1 \mathrm{~h}$ at room temperature in $5 \%$ nonfat dry milk/PBS-T. Finally, the target molecules were visualized through an enhanced chemiluminescence Western blotting detection system (GE Healthcare) on the X-ray film (GE Healthcare). The following primary and secondary antibodies used in this study were anti-matrin 3 (Santa Cruz Biotechnology, Santa Cruz, CA, USA), anti- $\beta$-actin (Sigma-Aldrich, St. Louis, MO, USA), and anti-IgG HRP antibodies (GE Healthcare). Immunoblotted bands were quantified using the Image Gauge (Fujifilm) after densitometric scanning of the films.

\section{Results}

\section{Requirement of matrin 3 for augmentation of gene expression by Xmn I fragment}

Figure 1A shows levels of luciferase induction during 24-h incubation in 2 types of cell following transient transfection of recombinant pGL3 plasmids carrying the Xmn I fragment. In Ac2F cells, pGL3-Xmn I augmented production of luciferase to approximately 5 times the control level. In Ac2F cells overexpressing matrin 3, pGL3 augmented production of luciferase up to approximately twice the control level and pGL3 Xmn I augmented production of luciferase to approximately 23 times the control level. Figure $1 \mathrm{~B}$ shows that 130 and $123 \mathrm{kDa}$ polypeptides detected by antirat matrin 3 antibodies were expressed approximately 3 -fold over the control in stable transfectants generated from Ac2F cells. In this context, given that matrin 3 is a phosphoprotein, the 130 and $123 \mathrm{kDa}$ polypeptides were presumed to be isoforms of matrin 3 [9].

\section{EMSA using the $X m n$ I fragment and nuclear extracts from wild matrin 3 highly expressed in Ac2F cells}

To determine whether matrin 3 actually binds to the Xmn I fragment, EMSA was performed using nuclear extracts from Ac2F cells and Ac2F cells highly expressing matrin 3 , and further using antirat matrin 3 antibody. The Xmn I fragment incubated with either control or matrin 3-highly expressing nuclear extract clearly retarded the signals (Figure 2, lanes 2 and 6) using electrophoretic conditions under which the Xmn I fragment alone migrates to near the bottom of a gel (lane 1). All of the radioactive signals shifted further following treatment of the reaction mixtures with anti-rat matrin 3 antibody (lanes 3-5 and 7-9), confirming that the retarded signals correctly reflect the DNA-binding properties of matrin 3. In contrast, anti-rat matrin 3 antibody showed no DNA binding activity (lane 10). 
Citation: Kamiuchi S, Fukaya M, Usui T, Iwata N, Okazaki M, et al. (2014) Matrin 3 Augments the Transcriptional Activity of an SV40 Promoter-

Page 3 of 5

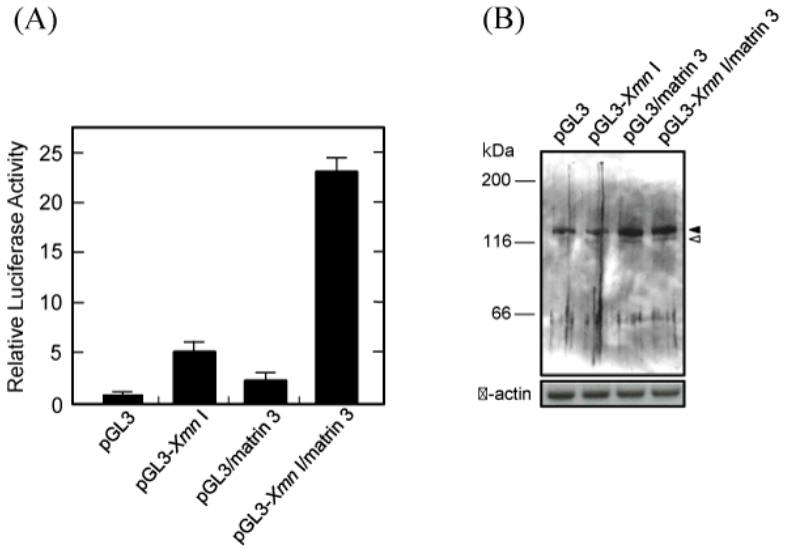

Figure 1: Induction of luciferase by transient transfection of recombinant plasmids with Xmn I fragment. (A) parental Ac2F cells or Ac2F cells overexpressing matrin 3 that received pGL3 and pGL3-Xmn I were cultured for $24 \mathrm{~h}$ and their lysates were prepared. Luciferase activities in Ac2F cells receiving pGL3 (relative to $\mathrm{pGL} 3$ ) are expressed as the mean value of 3 experiments $\pm \mathrm{SD}$. (B) Western blot analysis using anti-matrin 3 and $\beta$-actin antibodies were performed with whole cell extracts $(200 \mu \mathrm{g}$ protein) from parental Ac2F that received pGL3 and pGL3-Xmn I or Ac2F cells of matrin 3-stable transfectants generated from recombinant plasmids that received pGL3 (pGL3/matrin 3) and pGL3-Xmn I (pGL3-Xmn I/matrin 3). Filled and open arrowheads indicate 130 and $123 \mathrm{kDa}$ protein, respectively. Size of marker proteins is indicated along the left ordinate.

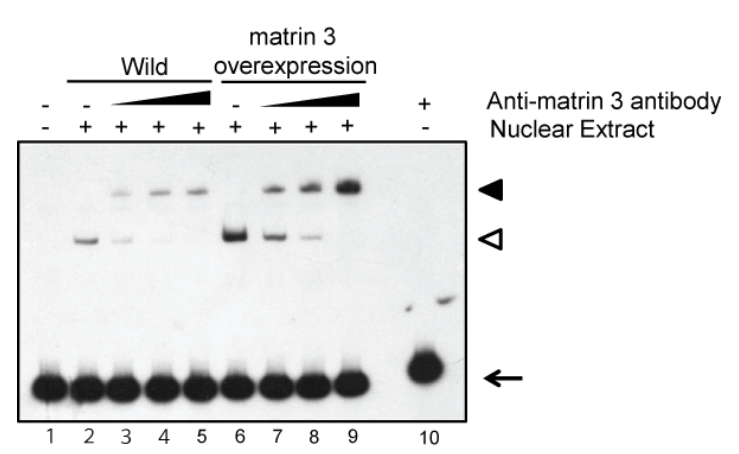

Figure 2: EMSA using $\left[{ }^{32} \mathrm{P}\right] X m n$ I fragments. Xmn I fragments alone (lanes 1 and 10 with $3 \mu \mathrm{g}$ anti-matrin 3 antibody) or Xmn I fragments incubated with nuclear extracts $(20 \mu \mathrm{g}$ protein $)$ from parental Ac2F (lanes 2-5 with $0,1,2$ and $3 \mu \mathrm{g}$ anti-matrin 3 antibody) or Ac2F cells of matrin 3-stable transfectants (lanes 6-9 with $0,1,2$ and $3 \mu \mathrm{g}$ anti-matrin 3 antibody) are resolved by PAGE followed by autoradiography. Filled and open arrowheads indicate supershift attempted with nuclear extract/anti-matrin 3 antibody and nuclear extract, respectively. Arrow indicates $\mathrm{Xmn}$ I fragments.

\section{Requirement of functional domain in matrin 3 for the augmentation of transcription}

Matrin 3 has several functionally confirmed motifs including NLS, NES, ZF1, ZF2, RB1, RB2, and MRS. To address the functional role of each motif in transcriptional activity, transient transfections with SV40 promoter-mediated luciferase gene with the Xmn I fragment were performed in Ac2F cells in which several deletion mutants of matrin 3 were overexpressed. Matrin 3 and its mutant proteins were expressed approximately 2-3 fold over the control in stable transfectants generated from Ac2F cells (data not shown). Figure 3 shows that pGL3-Xmn I augmented production of luciferase to approximately 4 times the control level in parental Ac2F cells, as described in Figure 1. Furthermore, in Ac2F cells overexpressing matrin 3, pGL3 augmented production of luciferase to approximately 2.5 times the control level and pGL3-Xmn I augmented production of luciferase to approximately 20 times the control level. Matrin 3 mutants devoid of ZF1 enhanced production to approximately $10 \%$ compared with the parental Ac2F cells. However, neither mutant devoid of ZF2 or ZF1/2 enhanced luciferase at all. In cells expressing mutants devoid of RB1, $\mathrm{RB} 2$, or RB1/2, luciferase activities decreased to a negligible level in parental cells. Matrin 3 mutants in which there were neither NES nor NLS including MRS did not augment luciferase activity.

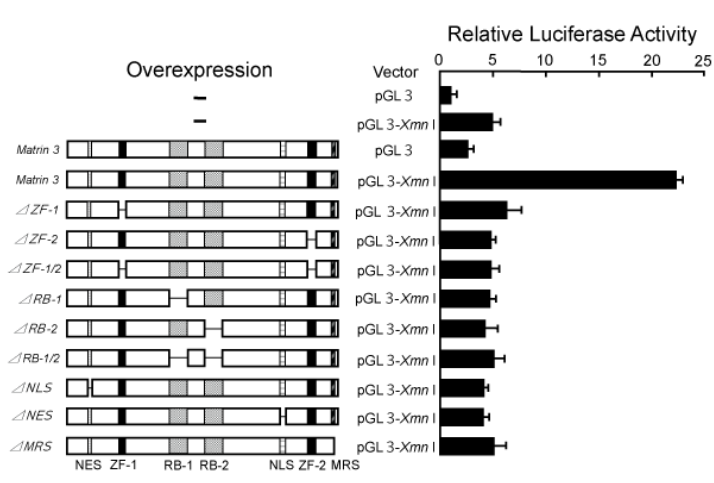

Figure 3: Requirement of a domain in matrin 3 for the augmentation of transcription. Lysates were prepared after $24 \mathrm{~h}$ incubation of Ac2F cells expressing wild-type or Matrin 3 for the transcriptional augmentation several type of deletion mutants of matrin 3 transfected with pGL3 promoter or pGL3-Xmn I (left panel) and luciferase activity was assayed (right panel).

\section{Discussion}

Our previous report described the principal properties of matrin 3 [3]. First, matrin 3 has several functionally confirmed motifs including NLS, NES, ZF1, ZF2, RB1, RB2, and MRS. Second, matrin 3 is localized not only in the nuclear scaffold but also in the soluble fraction in the nucleus, and in the cytoplasmic, microsomal and polysomal fractions of rat liver cells. Third, matrin 3 requires NLS and NES for subcellular trafficking, and ZF1 and ZF2 for translocation from soluble fraction to the nuclear scaffold fraction and therefore enabling the chromosomal association of matrin 3.

Fourth, matrin 3 is a phosphorylated protein and its phosphorylation status is altered in association with subcellular trafficking. These properties can be considered as the molecular basis of the diverse functions of matrin 3 . 
We also reported that Xmn I or several MAR/SAR fragments whose base unpairing appeared to play principal roles in binding to matrin 3 , when tethered upstream from the SV40 promoter increased reporter luciferase gene transcription [7].

In the present study, we demonstrated that the $X m n$ I fragment as a typical MAR/SAR augmented SV40 promoter-mediated luciferase gene transcription determined by transiently expressed luciferase activity (Figure 1). Furthermore, this segment in recombinant plasmids, can interact with matrin 3, and the enhanced interaction of $X m n$ I with overexpressed matrin 3 appeared to be required for augmentation of luciferase gene transcription (Figures 1 and 2). Furthermore, Figure 3 shows that all of the functional domains, not only ZF1 and ZF2 for DNA binding but also RB1 and RB2 for RNA binding, NLS and NES for subcellular trafficking, and MRS are independently required for augmentation of gene transcription in cells. In this context, the binding of matrin 3 to the Xmn I fragment may result in conditions under which proteins required to form the structural and functional basis of chromatins are more efficiently assembled. Furthermore, domain structure-mediated subcellular trafficking of matrin 3 may be involved in RNA metabolism in various ways.

It has been reported that chromatin may have the potential to change its configuration [10,11], as it is remodeled for transcription regulation. SATB1 regulates transcription activity and recruits chromatin-remodeling factors for region-specific histone modification [12-16], SATB2 activates gamma-globin genes by binding to MARs in their promoters and recruiting histone acetylase PCAF [17]. SAF-B suppresses transcription activity through a recombinant promoter constructs resembling the lysozyme 5' MAR promoter [18]. SMAR1, which interacts with chromatin modulators including HDAC1, and Sin3A causes chromatin condensation [19].

Histone deacetylase 1, a remodeling factor, interacts with the nuclear matrix [20] and with ARBP, a MAR-binding protein [21]. The expression of specific genes is enhanced by hnRNP-U by regulating mRNA stability [22].

Matrin 3, an abundant protein of the internal nuclear matrix, has been linked to a variety of functional events. Zeitz et al. have reported that matrin 3 interacts with 33 unique nuclear localized proteins and also revealed its propensity for self-association [23].

Recently, Skowronsk-Krqwczyk et al. reported that a homeodomain transcription factor regulates gene transcriptional programs through interaction with components of subnuclear structure such as the matrin 3-rich network [24].

Zhang and Carmichael reported that human inosine-specific RNA (I-RNA)-binidng protein p54nrb, a splicing factor PSF, and matrin 3 were co-purified from HeLa cells as a complex that binds hyperedited RNA, supposedly for anchoring it to the nuclear matrix [25]. Recently, it has been reported that matrin 3 that binds viral RNA is required for the Rev/RRE mediated nuclear export of unspliced HIV-1 RNAs [26-29], and it interacts in an RNA-dependent manner with several proteins with established roles in RNA processing, and maintains its interaction with RNA via its RNA recognition motif domain [30,31]. These data suggest that the cellular level of matrin 3 , known to be highly regulated, modulates the stability of a group of gene transcripts.

Animal and plant genomes carry MAR/SAR, which also interact with the nuclear matrix. MAR/SAR segments are reportedly expected to be present at both up and downstream boundaries of nuclear genes
[32] and are predicted to be distributed throughout the genome [33]. MAR/SAR positioned in the vicinity of a promoter can reportedly augment transcription of a reporter gene, when the recombinant construct is integrated into the genome of the host animal, suggesting that MAR/SAR as cis-acting elements could boost the expression of recombinant proteins from cultured cells. Given that gene transfer in eukaryotic cells and organisms suffers from epigenetic effects that result in low or unstable transgene expression and high clonal variability, use of epigenetic regulators such as MAR/SAR is a promising approach to alleviate such unwanted effects [34]. Thus this study also suggests new strategies for the application of potent epigenetic regulators, MAR/SAR with matrin 3 toward high and stable transgene expression for research, therapeutic production, or genebased therapies.

\section{Acknowledgments}

This study was supported in part by a Grant-in-Aid from the Ministry of Education, Culture and Science of Japan (15602002, 18510173). The authors would like to thank Enago (www.enago.jp) for the English language review.

\section{References}

1. Mirkovitch J, Gasser SM, Laemmli UK (1987) Relation of chromosome structure and gene expression. Philos Trans R Soc Lond B Biol Sci 317: 563-574.

2. Ma H, Siegel AJ, Berezney R (1999) Association of chromosome territories with the nuclear matrix. Disruption of human chromosome territories correlates with the release of a subset of nuclear matrix proteins. J Cell Biol 146: 531-542.

3. Hibino Y, Usui T, Morita Y, Hirose N, Okazaki M, et al. (2006) Molecular properties and intracellular localization of rat liver nuclear scaffold protein P130. Biochim Biophys Acta 1759: 195-207.

4. Belgrader P, Dey R, Berezney R (1991) Molecular cloning of matrin 3. A 125-kilodalton protein of the nuclear matrix contains an extensive acidic domain. J Biol Chem 266: 9893-9899.

5. Ikeda Y, Nakamura K, Iwakami N, Hibino Y, Sugano N (1990) Base sequences of highly repetitive components in nuclear DNAs from rat liver and rat-ascites hepatoma. Cancer Lett 55: 201-208.

6. Dickinson LA, Joh T, Kohwi Y, Kohwi-Shigematsu T (1992) A tissuespecific MAR/SAR DNA-binding protein with unusual binding site recognition. Cell 70: 631-645.

7. Hibino Y, Ohzeki H, Sugano N, Hiraga K (2000) Transcription modulation by a rat nuclear scaffold protein, P130, and a rat highly repetitive DNA component or various types of animal and plant matrix or scaffold attachment regions. Biochem Biophys Res Commun 279: 282-287.

8. Hibino Y, Nakamura K, Tsukada S, Sugano N (1993) Purification and characterization of nuclear scaffold proteins which bind to a highly repetitive bent DNA from rat liver. Biochim Biophys Acta 1174: 162-170.

9. Hibino Y, Ohzeki H, Hirose N, Sugano N (1998) Involvement of phosphorylation in binding of nuclear scaffold proteins from rat liver to a highly repetitive DNA component. Biochim Biophys Acta 1396: 88-96.

10. Zink D, Cremer T (1998) Cell nucleus: chromosome dynamics in nuclei of living cells. Curr Biol 8: R321-324.

11. Fry CJ, Peterson CL (2001) Chromatin remodeling enzymes: who's on first? Curr Biol 11: R185-197.

12. Kohwi-Shigematsu T, Maass TK, Bode J (1997) A thymocyte factor SATB1 suppresses transcription of stably integrated matrix-attachment region-linked reporter genes. Biochemistry 36: 12005-12010.

13. Liu J, Bramblett D, Zhu Q, Lozano M, Kobayashi R, et al. (1997) The matrix attachment region-binding protein SATB1 participates in 
Citation: Kamiuchi S, Fukaya M, Usui T, Iwata N, Okazaki M, et al. (2014) Matrin 3 Augments the Transcriptional Activity of an SV40 PromoterMediated Luciferase Gene with a Highly Repetitive DNA Component. J Mol Genet Med 8: 146. doi:10.4172/1747-0862.1000146

Page 5 of 5

negative regulation of tissue-specific gene expression. Mol Cell Biol 17: 5275-5287.

14. Cai S, Han HJ, Kohwi-Shigematsu T (2003) Tissue-specific nuclear architecture and gene expression regulated by SATB1. Nat Genet 34: $42-51$.

15. Cai S, Lee CC, Kohwi-Shigematsu T (2006) SATB1 packages densely looped, transcriptionally active chromatin for coordinated expression of cytokine genes. Nat Genet 38: 1278-1288.

16. Kohwi-Shigematsu T, Kohwi Y, Takahashi K, Richards HW, Ayers SD, et al. (2012) SATB1-mediated functional packaging of chromatin into loops. Methods 58: 243-254.

17. Zhou LQ, Wu J, Wang WT, Yu W, Zhao GN, et al. (2012) The AT-rich DNA-binding protein SATB2 promotes expression and physical association of human $(\mathrm{G}) \gamma$ - and (A) $\gamma$-globin genes. J Biol Chem 287: 30641-30652.

18. Nayler O, Strätling W, Bourquin JP, Stagljar I, Lindemann L, et al. (1998) SAF-B protein couples transcription and pre-mRNA splicing to SAR/MAR elements. Nucleic Acids Res 26: 3542-3549.

19. Nakka KK, Chattopadhyay S (2010) Modulation of chromatin by MARs and MAR binding oncogenic transcription factor SMAR1. Mol Cell Biochem 336: 75-84.

20. Li W, Chen HY, Davie JR (1996) Properties of chicken erythrocyte histone deacetylase associated with the nuclear matrix. Biochem J 314 631-637.

21. Weitzel JM, Buhrmester H, Strätling WH (1997) Chicken MAR-binding protein ARBP is homologous to rat methyl-CpG-binding protein MeCP2. Mol Cell Biol 17: 5656-5666.

22. Yugami M, Kabe Y, Yamaguchi Y, Wada T, Handa H (2007) hnRNP-U enhances the expression of specific genes by stabilizing mRNA. FEBS Lett 581: 1-7.

23. Zeitz MJ, Malyavantham KS, Seifert B, Berezney R (2009) Matrin 3: chromosomal distribution and protein interactions. J Cell Biochem 108 125-133.
24. Skowronska-Krawczyk D, Ma Q, Schwartz M, Scully K, Li W, et al. (2014) Required enhancer-matrin-3 network interactions for a homeodomain transcription program. Nature 514: 257-261.

25. Zhang Z, Carmichael GG (2001) The fate of dsRNA in the nucleus: a p54(nrb)-containing complex mediates the nuclear retention of promiscuously A-to-I edited RNAs. Cell 106: 465-475.

26. Kula A, Guerra J, Knezevich A, Kleva D, Myers MP, et al. (2011) Characterization of the HIV-1 RNA associated proteome identifies Matrin 3 as a nuclear cofactor of Rev function. Retrovirology 8: 60 .

27. Yedavalli VS, Jeang KT (2011) Matrin 3 is a co-factor for HIV-1 Rev in regulating post-transcriptional viral gene expression. Retrovirology 8: 61.

28. Dayton AI (2011) Matrin 3 and HIV Rev regulation of mRNA. Retrovirology 8: 62.

29. Kula A, Gharu L, Marcello A (2013) HIV-1 pre-mRNA commitment to Rev mediated export through PSF and Matrin 3. Virology 435: 329-340.

30. Salton M, Elkon R, Borodina T, Davydov A, Yaspo ML, et al. (2011) Matrin 3 binds and stabilizes mRNA. PLoS One 6: e23882.

31. Yamazaki F, Kim HH, Lau P, Hwang CK, Iuvone PM, et al. (2014) pY RNA1-s2: a highly retina-enriched small RNA that selectively binds to Matrin 3 (Matr3). PLoS One 9: e88217.

32. Schübeler D, Mielke C, Maass K, Bode J (1996) Scaffold/matrix-attached regions act upon transcription in a context-dependent manner. Biochemistry 35: 11160-11169.

33. Girod PA, Nguyen DQ, Calabrese D, Puttini S, Grandjean M, et al. (2007) Genome-wide prediction of matrix attachment regions that increase gene expression in mammalian cells. Nat Methods 4: 747-753.

34. Harraghy N, Buceta M, Regamey A, Girod PA, Mermod N (2012) Using matrix attachment regions to improve recombinant protein production. Methods Mol Biol 801: 93-110. 